\title{
Online atlasing using an iterative centroid *
}

\author{
Antoine Legouhy ${ }^{1}$, Olivier Commowick ${ }^{1}$, François Rousseau ${ }^{2}$, and Christian \\ Barillot $^{1}$ \\ 1 Univ Rennes, INRIA, CNRS, INSERM, IRISA UMR 6074, Empenn ERL U-1228, \\ F-35000, Rennes, France \\ 2 IMT Atlantique, LaTIM U1101 INSERM, UBL, Brest, France
}

\begin{abstract}
Online atlasing, i.e. incrementing an atlas with new images as they are acquired, is key when performing studies on databases very large or still being gathered. We propose to this end a new diffeomorphic online atlasing method without having to perform again the atlasing process from scratch. New subjects are integrated following an iterative procedure gradually shifting the centroid of the images to its final position, making it computationally cheap to update regularly an atlas as new images are acquired (only needing a number of registrations equal to the number of new subjects). We evaluate this iterative centroid approach through the analysis of the sharpness and variance of the resulting atlases, and the transformations of images, comparing their deviations from a conventional method. We demonstrate that the transformations divergence between the two approaches is small and stable and that both atlases reach equivalent levels of image quality.
\end{abstract}

\section{Introduction}

An atlas is a generic term covering several uses. Overall, it corresponds to an average model of the brain both in terms of shape and intensity. It is therefore a powerful tool to understand brain variability, to compute statistics on populations [14] or to segment regions [3. Ideally, atlases are made from a large number of subjects by combining their images through registration and transformation composition which can be computationally quite costly.

Among the many available atlas construction methods, Guimond et al. [8] proposed to register the subjects onto an arbitrary image, followed by an unbiasing and averaging step, leading to an atlas that becomes the new reference image. By iterating the process, the atlas becomes less and less biased by the initial reference image. Joshi et al. and Rohlfing et al. 9,12 among others focused on another atlas construction class, called template free approaches, working with groupwise registration. Although those methods have the advantage of being unbiased by construction, their computational cost can be prohibitive.

\footnotetext{
* The research leading to these results has been supported by the ANR MAIA project, grant ANR-15-CE23-0009 of the French National Research Agency (http: //recherche.imt-atlantique.fr/maia) and La Région Bretagne.
} 
Historically databases used for atlas construction were small (about 50 to 100 subjects at most). However, there is a recent trend for the creation of large databases, out of which great atlases depicting the variability of the population across the life span could be inferred. Among these databases, the human connectome project (HCP, now completed) is made of around 1200 subjects of the same age range [7. Even bigger databases are currently being constituted from more subjects (up to 100,000 in the UK biobank [11]) or across the life span (HCP lifespan) on which researchers already wish to start studies. This causes a problem as the databases are still being gathered and thus the atlas needs to be updated. With current methods, the atlas needs to be recomputed each time new acquisitions are added which is very costly. New methods are therefore needed to allow online atlasing, i.e. the capacity of incrementing atlases with new images as they come without having to perform again the whole atlas construction, as opposed to the above-mentioned direct atlasing.

We propose a new method that considers atlasing from a different angle. Inspired by the notion of centroid for a set of points in a Euclidean space and the way it can be constructed following an iterative procedure, Cury et al. [6] derived a diffeomorphic atlas construction method working on surfaces. We propose here a generalization of this iterative centroid method for image atlasing where the centroid (atlas) is updated gradually as new images are incorporated. The online aspect of this technique has several advantages: when a new images arrives, its integration does not necessitate to restart the atlasing process from scratch. Also, it only requires one registration per new image making it computationally cheap. Benefitting from the log-Euclidean framework for diffeomorphisms from Arsigny et al. 1], the method allows the production of a diffeomorphic atlas unbiased up to a linear transformation (rigid or affine at the choosing of the user).

We first introduce the iterative centroid method and its specificities for image atlasing in Section 2. Then in Section 3, we present experiments comparing how the atlases built using direct atlasing (here a modified Guimond et al. method [10]) and with our method differ from each other as they include more and more subjects. We demonstrate with three different metrics that the iterative centroid and direct atlases diverge only by a small amount in terms of transformations and that their intensity based features do not vary.

\section{Method}

\subsection{Theoretical background}

Considering the registration of an image $J$ onto an image $I$, we denote by $\tilde{J}$ the registered image and $T$ the transformation operating on coordinates $x$ used to resample $J$ onto $I: I(x) \sim \tilde{J}(x)=J \circ T(x)$. Assuming that $T$ lives in a connected Riemannian manifold, we consider $\gamma(t)$ the geodesic between $I d$ and $T$ such that $\gamma(0)=I d$ and $\gamma(1)=T$. In this framework (named large deformation diffeomorphic metric mapping - LDDMM), the deformation $T$ is parameterized by a time varying vector field integrated over time (the geodesic $\gamma$ ). 
Diffeomorphisms are widely used in non linear registration because of their interesting properties: differentiability, bijectivity and differentiability of the inverse map. As the LDDMM is a computationally intensive framework, Arsigny et al. 1] presented a log-Euclidean framework to compute statistics on diffeomorphisms parameterized by a time independent i.e. stationary velocity field (SVF). For such diffeomorphisms, operations are defined as their Euclidean counterparts on the SVFs. One of interest is the power of a diffeomorphism defined as $T^{\alpha}=\gamma(\alpha)=\exp (\alpha \log (T))$. In the following, all non linear transformations are assumed to be diffeomorphisms parameterized with SVFs.

Additionally, given 2 SVFs $v$ and $w$, it is possible to compute the logarithm of their composition while avoiding a very costly logarithm computation. This is made possible by the Baker-Campbell-Hausdorff $(\mathrm{BCH})$ formula stating that in a BCH-Lie group, for $v$ and $w$ small enough, $\log (\exp (v) \circ \exp (w))$ can be approximated as a series of Lie brackets:

$$
\log (\exp (v) \circ \exp (w)) \approx \operatorname{BCH}(v, w)=v+w+\frac{1}{2}[v, w]+\ldots
$$

Where $[v, w](x)=\operatorname{Jac}(v)(x) \cdot w(x)-\operatorname{Jac}(w)(x) \cdot v(x)$. It has been shown in 2113. that the use of the $\mathrm{BCH}$ formula is well suited for diffeomorphisms parameterized as SVFs. To simplify notations, unless specified otherwise, the composition of two transformations associated to two SVFs $v$ and $w$ is performed via the $\mathrm{BCH}$ : $\exp (v) \circ \exp (w)=\exp (\operatorname{BCH}(v, w))$.

\subsection{Iterative centroid atlas construction}

In a Euclidean space $\Omega$, the centroid $b_{n}$ of a set of points $\left\{x_{1}, \ldots, x_{n}\right\}$ is defined as $b_{n}=\operatorname{argmin}_{y \in \Omega} \sum_{i=1}^{n}\left\|y-x_{i}\right\|^{2}$. A direct solution is $\frac{1}{n} \sum_{i=1}^{n} x_{i}$ but an iterative approach is also imaginable. Starting from $b_{1}=x_{1}$, we can use the recursion:

$$
b_{k+1}=\frac{k}{k+1} b_{k}+\frac{1}{k+1} x_{k+1}
$$

As depicted in [6], one can extend this formulation to the Riemannian case for surfaces by using the generalization of the notion of straight line through geodesics. The updated centroid $b_{k+1}$, in that case, is on the geodesic linking $b_{k}$ and $x_{k+1}$, at $\frac{1}{k+1}$ times the distance from $b_{k}$.

We define our iterative centroid atlasing, following the same spirit, on images and diffeomorphisms parameterized by SVFs. Thereafter we assume that the registration of an image $I$ onto an atlas $A$ provides two transformations $L$ and $T$, where $L$ is linear and $T$ is a diffeomorphism, so that $A \sim I \circ L \circ T$. Generalizing Eq. 2 to a set of images $\left\{I_{1}, \ldots, I_{n}\right\}$, we start from $A_{1}=I_{1}$ and get:

$$
A_{k+1}=\frac{k}{k+1} A_{k} \circ T_{k+1}^{-\frac{1}{k+1}}+\frac{1}{k+1} I_{k+1} \circ L_{k+1} \circ T_{k+1}^{\frac{k}{k+1}}
$$

The atlasing scheme is illustrated Fig. 1. We apply to $A_{k}$ a transformation situated at $\frac{1}{k+1}$ of the distance from the identity to $T_{k+1}^{-1}$ along the geodesic. We 
also apply to $I_{k+1}$, in addition to the linear transformation, a transformation situated at $\frac{k}{k+1}$ of the distance from the identity to $T_{k+1}$ along the geodesic. Also, $A_{k}$ is made from $k$ images so a weight $\frac{k}{k+1}$ is affected to its intensities while $I_{k+1}$ intensities get a weight $\frac{1}{k+1}$. At the end, those two images are transformed and weighted according to their contribution to the atlas.

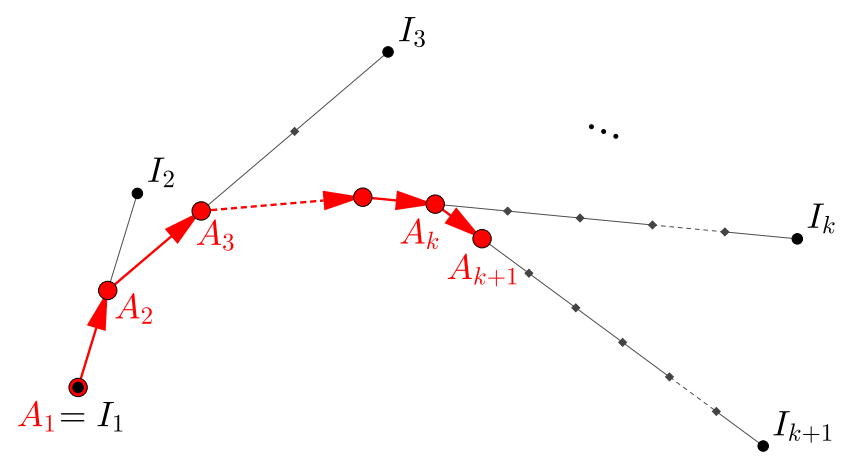

Fig. 1. Shift of the centroid as new images are incorporated.

Unlike [6], we are dealing with images and therefore interpolations on a voxel grid to reconstruct a new image after a transformation. This resampling step has to be avoided as much as possible to keep a maximum of details. To this end it is preferable not to use $A_{k}$ (already a resampled image) when constructing $A_{k+1}$, but instead to operate on the initial images using transformation compositions such that each image undergoes only one resampling. This is achieved by expressing the sequence $\left(A_{k}\right)_{k \in \mathbb{N}}$ using only the initial images $\left\{I_{j}, j=1, \ldots, k\right\}$. Rearranging Eq. 3, the following formulation emerges:

$$
A_{k+1}=\frac{1}{k+1}\left(\sum_{j=1}^{k}\left(I_{j} \circ L_{j} \circ \Theta_{j, k} \circ T_{k+1}^{-\frac{1}{k+1}}\right)+I_{k+1} \circ L_{k+1} \circ T_{k+1}^{\frac{k}{k+1}}\right)
$$

Where $\theta_{j, k}$ is the non linear part of the transformation bringing $I_{j}$ onto $A_{k}$. There are $k$ compositions using $\mathrm{BCH}$ to perform at iteration $k$ which correspond to the update of the transformations $\theta_{j, k}$ for $j \leq k$.

Initialization: One can start at iteration 1 by initiating $A_{1}=I_{1}$ and $\theta_{1,1}=I d$. However, a very interesting point of the method is the possibility to complement an already existing atlas $A_{p}$ constituted of $p$ images as long as it can be written as $A_{p}=\frac{1}{p} \sum_{j=1}^{p} I_{j} \circ L_{j} \circ T_{j}$. To do so, we simply assign $\theta_{j, p}=T_{j}, \forall j \leq p$.

Iterative procedure: At the end of iteration $k$, we have, for each $j \leq k$, transformations $\theta_{j, k}$ that map images $I_{j}$ onto the atlas $A_{k}: A_{k} \sim I_{j} \circ L_{j} \circ \theta_{j, k}$. 
To build $A_{k+1}$ from these, we do the following: 1- we register $I_{k+1}$ onto $A_{k}$ : $A_{k} \sim I_{k+1} \circ L_{k+1} \circ T_{k+1}$ (one registration) ; 2 - for $j \leq k$, we compute the updated $\theta_{j, k+1}$ as $\theta_{j, k+1}=\theta_{j, k} \circ T_{k+1}^{-\frac{1}{k+1}}(k \mathrm{BCH}) ; 3$ - we assign $\theta_{k+1, k+1}=T_{k+1}^{\frac{k}{k+1}} ;$ and finally 4 - we build $A_{k+1}=\frac{1}{k+1} \sum_{j=1}^{k+1} I_{j} \circ L_{j} \circ \theta_{j, k+1}$.

\subsection{Unbiased atlas construction}

So far, we have only assumed that the registration produced a linear and a non linear part. Going in more details, let us now assume that this registration can be written $A \sim I \circ R \circ S \circ D$ where: $R \circ S$ is an affine transformation decomposed into a rigid part $R$ and a stretching part $S$, and $D$ is a diffeomorphism. Herebefore, by taking $L=R \circ S$ and $T=D$ i.e. $L$ encapsulating all the linear transformations and $T$ only local displacements, the method will lead - similarly to 8 - to an atlas unbiased up to an affine transformation. On the other hand, if we take $L=R$ and $T=S \circ D$ i.e. $L$ only considering rigid motion while $T$ encapsulating both growth and local displacements, the method will lead to an atlas unbiased up to a rigid transformation (useful typically for longitudinal studies).

\section{Experiments and results}

\subsection{MRI database}

For our experiments, we have used T1 weighted preprocessed images (brain extracted) from 100 subjects randomly chosen among the Human Connectome Project (HCP) database (for more details, see Van Essen et al. [7]). The size of the images is $260 \times 311 \times 260$ with a voxel size of $0.7 \mathrm{~mm}$ isotropic.

\subsection{Implementation details}

Registration Our method is agnostic to the registration used. We performed registrations in two steps using symmetric block matching algorithms [5] available in Anima!

1. An affine registration that outputs a transformation matrix $B$ which is decomposed through polar decomposition into a rigid part $R$ and a stretching part $S$ as depicted in Legouhy et al. [10].

2. A diffeomorphic registration that directly outputs the SVF of the diffeomorphism $\log (D)$ linking the two images. This allows to take advantage of the log-Euclidean framework without logarithm computation [13/2].

We chose to compute atlases up to a rigid transformation. Therefore, the linear transformation $L$ is rigid: $L=R$ and the non linear transformation is the combination of stretching and local displacements: $T=S \circ D$.

\footnotetext{
${ }^{1}$ Anima: Open source software for medical image processing from the Empenn team. https://github.com/Inria-Visages/Anima-Public - RRID:SCR_017017
} 
Transformation composition Compositions of two diffeomorphisms are performed on their SVFs using order $2 \mathrm{BCH}$. To compose a linear transformation $L$ and a SVF, we first apply $\log (L)$ to the real coordinates of an image grid to transform it into an SVF and then compose using order $2 \mathrm{BCH}$.

\subsection{Comparison between iterative centroid and direct atlasing}

In the following experiments, we evaluate the quality of the iterative centroid atlas and its divergence with respect to direct atlasing, here a method developed in Legouhy et al. [10] available in Anima-Scripts ${ }^{2}$ based on Guimond et al. 8]. For both methods, we used the same registration and composition tools with the same parameters. Starting from an atlas built with 50 images using direct atlasing, we added one by one the 50 other images using our online atlasing. We then compared the direct atlases computed at several steps with the online ones using three metrics.

Divergence Both methods output linear and non linear transformations (denoted $T_{j}$ for online atlasing and $\tilde{T}_{j}$ for direct atlasing) mapping each image onto the atlas. We first propose to evaluate the divergence between the two atlases $A$ and $\tilde{A}$ by an image $\delta$ defined from the transformations: $\delta(A, \tilde{A})=$ $\frac{1}{n} \sum_{j=1}^{n} \sqrt{\sum_{d=1}^{3}\left(T_{j, d}-\tilde{T}_{j, d}\right)^{2}}$ where $d$ indexes the three components in space. This measure has the advantage of being agnostic to edge issues that can happen in intensities comparisons.

Fig. 2 presents a qualitative view of the evolution of the differences between the direct and online atlases with respect to the number of subjects. We observe that most of the differences increase with the number of added subjects and occur in the cortical areas while central structures of the brain are spared. Fig. 3 gives quantitative plots over the brain of the divergence measures. It suggests that the divergence tends to grow (from a median of $0.777 \mathrm{~mm}$ for 60 images, to $1.095 \mathrm{~mm}$ for 100) but at a slow pace that decreases $(+0.153 \mathrm{~mm}$ between 60 and 70 images but only $+0.025 \mathrm{~mm}$ between 90 and 100).

Iconic evaluations The second part of the evaluation assesses the quality of the atlases based on image intensities. We evaluate first the atlas standard deviation across subject images after transformation on the atlas, the transformations being constructed differently upon the atlasing method. This measure allows to quantify how well the images are aligned after atlas construction (the lower the standard deviation, the better): $\operatorname{sdis}(A)=\sqrt{\frac{1}{n} \sum_{i=j}^{n}\left(I_{j} \circ L_{j} \circ T_{j}-A\right)^{2}}$. For better interpretation, we normalized sdis by the average of the intensities of the atlases from the two methods to create $\operatorname{sdis}_{n}$.

\footnotetext{
${ }^{2}$ Anima-Scripts: Open source scripts using Anima software for medical image processing from the Empenn team. https://github.com/Inria-Visages/ Anima-Scripts-Public - RRID:SCR_017072
} 
In addition, we propose a measure of the sharpness of the atlases. Given $N(i)$, a patch around a voxel $i$ of an image $A$, we define a local measure of the sharpness at voxel $i$, sha $(A(i))$, as the standard deviation of the patch normalized by its average: $\operatorname{sha}(A(i))=\operatorname{sd}_{N(i)}(A) / \operatorname{mean}_{N(i)}(A)$. In our experiments, we chose a patch of diameter 5 voxels. We present in Fig. 4 a quantitative view over voxels inside the brain mask of these two iconic measures with respect to the number of images in the atlas. These results show that both methods output atlases that are very similar in terms of image quality. The sharpness remains equivalent for both methods independently of the number of images added. sdis $_{n}$ increases only between 60 and 70 images and is then stable, but the evolution and values are the same for both atlas construction methods.

\section{Conclusion}

We have presented an online atlasing method that allows the incorporation of new images into an existing atlas without having to restart the atlasing process from the beginning. Based on an iterative centroid process, this approach only necessitates one registration per new image. We observed that the divergence between our method and direct atlasing using the same tools is localized in cortical areas and tends to grow but at a slow and decreasing pace. Also, the obtained atlases from both approaches have shown no differences in terms of image quality. The trend being at large, growing databases, the proposed online atlasing
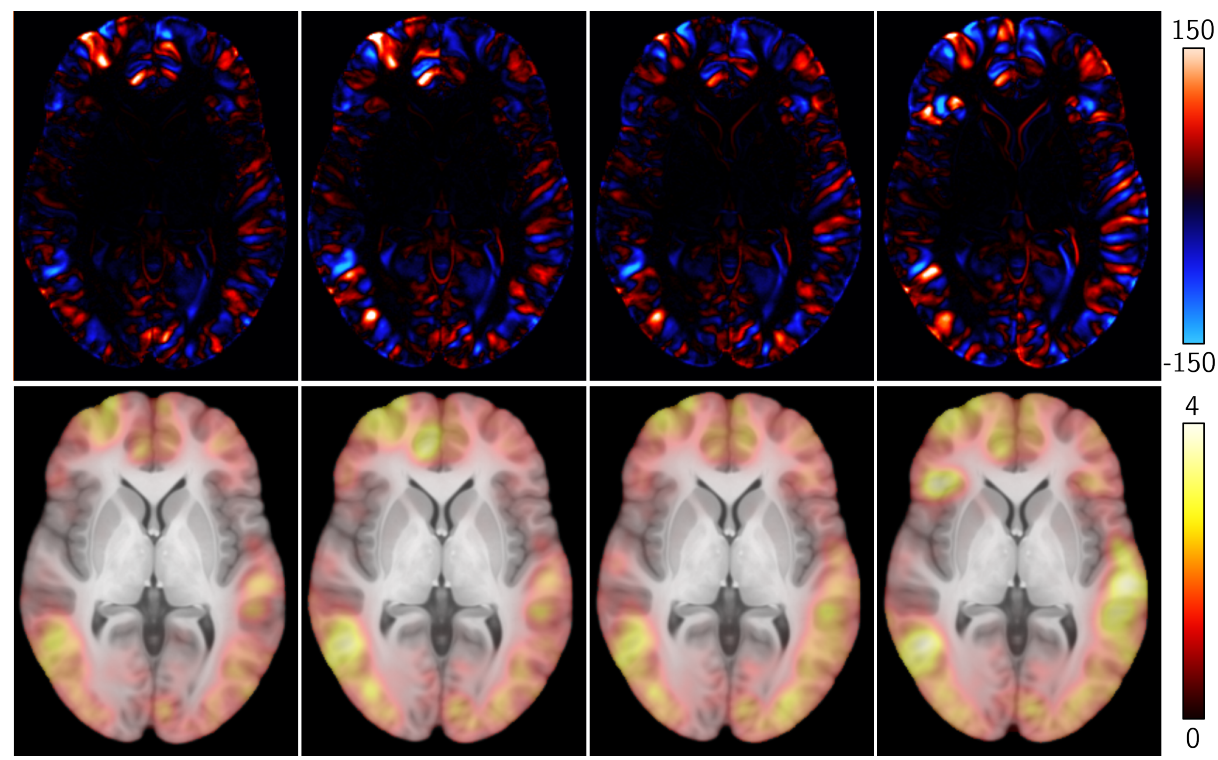

Fig. 2. From left to right atlases with $60,70,80$ and 100 images. Top: intensity differences between iterative centroid and direct atlases. Bottom: atlases from iterative centroid method overlaid with divergence measure $\delta$. 


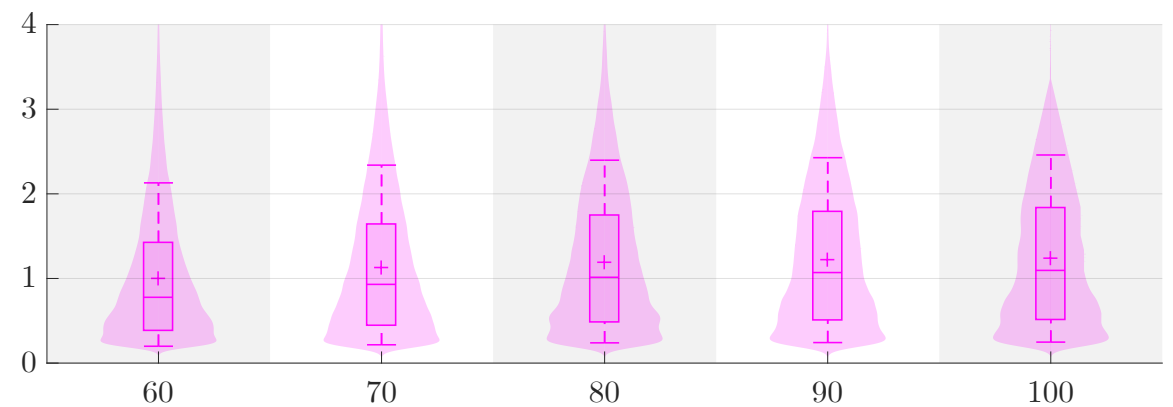

Fig. 3. Boxplots and violin plots of the divergence $\delta$ between the atlases (restrained to the union of the masks) with $60,70,80,90$ and 100 images.

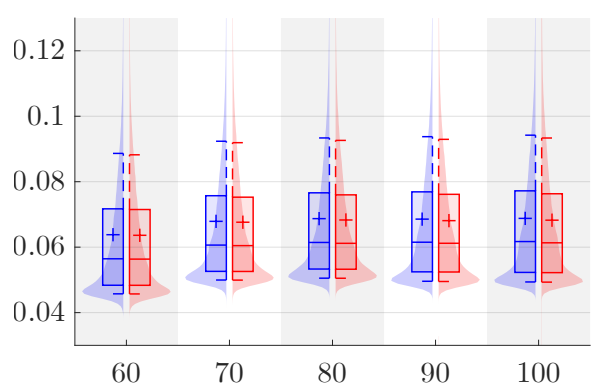

(a)

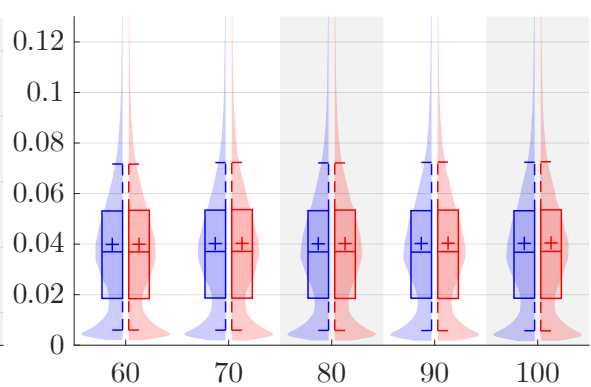

(b)

Fig. 4. Boxplots and violin plots of inter-subjects normalized standard deviations sdis ${ }_{n}$ (a) and sharpness sha (b) for atlases (restrained to the union of the masks) with 60, 70, 80, 90 and 100 images. Blue: iterative centroid. Red: direct atlasing.

method offers an interesting tool to update an atlas at reasonable computational cost as new images arrive. Finally, we derived a construction method up to either an affine or a rigid transformation. This makes the method especially eligible for longitudinal atlasing as a future work, simply by adapting the weight of each image in Eq. 4

\section{References}

1. Arsigny, V., Commowick, O., Pennec, X., Ayache, N.: A Log-Euclidean Framework for Statistics on Diffeomorphisms. In: MICCAI. pp. 924-931 (2006)

2. Bossa, M., Hernandez, M., Olmos, S.: Contributions to 3D Diffeomorphic Atlas Estimation: Application to Brain Images. In: MICCAI. pp. 667-674 (2007)

3. Cabezas, M., Oliver, A., Lladó, X., Freixenet, J., Cuadra, M.B.: A review of atlasbased segmentation for magnetic resonance brain images. Computer Methods and Programs in Biomedicine 104(3), e158 - e177 (2011)

4. Commowick, O., Wiest-Daesslé, N., Prima, S.: Automated diffeomorphic registration of anatomical structures with rigid parts: application to dynamic cervical MRI. In: MICCAI. pp. 163-170 (2012) 
5. Commowick, O., Wiest-Daesslé, N., Prima, S.: Block-matching strategies for rigid registration of multimodal medical images. In: ISBI. pp. 700-703 (2012)

6. Cury, C., Glaunès, J.A., Colliot, O.: Diffeomorphic Iterative Centroid Methods for Template Estimation on Large Datasets. Geometric Theory of Information Chapter 10, 273-299 (2014)

7. Essen, D.V., Ugurbil, K., et al.: The human connectome project: A data acquisition perspective. NeuroImage 62(4), 2222 - 2231 (2012)

8. Guimond, A., Meunier, J., Thirion, J.P.: Average brain models: A convergence study. Computer vision and image understanding (2000)

9. Joshi, S., Davis, B., Jomier, M., Gerig, G.: Unbiased diffeomorphic atlas construction for computational anatomy. NeuroImage 23, S151-S160 (2004)

10. Legouhy, A., Commowick, O., Rousseau, F., Barillot, C.: Unbiased longitudinal brain atlas creation using robust linear registration and log-Euclidean framework for diffeomorphisms. In: ISBI. pp. 1038-1041. IEEE (2019)

11. Miller, K.L., et al.: Multimodal population brain imaging in the UK biobank prospective epidemiological study. Nature Neuroscience 19, 1523 EP - (2016)

12. Rohlfing, T., Zahr, N.M., et al.: The SRI24 Multichannel Atlas of Normal Adult Human Brain Structure. Human Brain Mapping 31, 798-819 (2010)

13. Vercauteren, T., Pennec, X., et al.: Symmetric Log-Domain Diffeomorphic Registration: A Demons-Based Approach. In: MICCAI. pp. 754-761 (2008)

14. Whitcher, B., Wisco, J.J., et al.: Statistical group comparison of diffusion tensors via multivariate hypothesis testing. MRM 57(6), 1065-1074 (2007) 